\title{
Nutritional Status and Informational Needs for Patients with Liver Cirrhosis
}

Lamiaa Zaki Ahmed ${ }^{1}$, Nadia Mohamed Taha ${ }^{2}$, Mohamed El-khashab ${ }^{3}$ and Eman Elsayed Hussein ${ }^{4}$

${ }^{1}$ B.Sc. Nursing, Faculty of Nursing, Zagazig University, Egypt.

${ }^{2}$ Medical Surgical Nursing, Faculty of Nursing, Zagazig University, Egypt.

${ }^{3}$ Tropical medicine Department, Faculty of medicine, Zagazig University, Egypt.

${ }^{4}$ Medical Surgical Nursing, Faculty of Nursing, Zagazig University,Egypt.

Corresponding Author

Lamiaa Zaki Ahmed

Mobile:

$+201096652481$

E mail:

lamiaazaki631@yahoo .com

Key words:

liver cirrhosis,

Malnutrition,

informational needs
Background and study aim: Malnutrition is as an important complication of liver cirrhosis with prognostic implications; a marked knowledge gap exists concerning the information needs of liver cirrhosis patients. The aim of this study is to assess nutritional status and informational needs for patients with liver cirrhosis.

Subjects and Methods: A descriptive exploratory design was used in this study. The present study conducted in tropical medicine and gastroenterology units at Zagazig University Hospitals, A purposive sample of 115 patients, The study lasted from the beginning of October 2016 to the end of May 2017 .Three tools were used for collection of data, first tool was a structured interview questionnaire consisted of personal characteristics of patients, second tool was nutritional assessment form, third tool was informational needs questionnaire.

Results: The study findings revealed that more two third of studied patients were in

\section{INTRODUCTION}

Hepatitis $\mathrm{C}$ virus is one of the chief causes of chronic liver disease. Hepatitis $\mathrm{C}$ related liver disease encompasses a wide spectrum ranging from chronic hepatitis $\mathrm{C}$ to compensated cirrhosis and eventually to decompensated cirrhosis and hepatocellular carcinoma [1]. Cirrhosis is the final common pathway for the majority of liver diseases, and is a complex chronic condition that causes population mortality rates of approximately $5-10$ per 100,000 person-years worldwide [2]. Nutrition is an integral part of health maintenance. Progressive deterioration of nutritional the age group of less than 60 years with mean age $53.4 \pm 9.3$ years, the majority of studied patients were in malnutrition (73.9\%). By Subjective Global Assessment (SGA), there are strong correlation between SGA and Child Pugh score, the most important informational needs among the studied patients were medical domains $84.4 \%$. In contrast, psychological domains achieved the least important $27.0 \%$.

Conclusion: It can be concluded that patients with liver cirrhosis are suffering from malnutrition and nutritional deficiencies, and also patient with liver cirrhosis had different levels of informational needs. The most prioritized informational needs for patients with liver cirrhosis was Medical domain whereas the least priority was given to the Psychological domain. It also shows that information needs differ based on some socio-demographic and clinical characteristics and physical condition.

status has been associated with poor outcome in cirrhotic patients [3]. Proteinenergy malnutrition (PEM) is highly prevalent in patients with liver disease and leads to serious repercussions on the general state, having a direct impact on cirrhotic patient prognosis, deteriorating liver function, adversely affecting the clinical evolution [4]. The prevalence of malnutrition in decompensated cirrhosis ranges from $60 \%-100 \%$, while $20 \%-30 \%$ of patients with compensated cirrhosis are also malnourished [5]. The pathogenesis of malnutrition in chronic liver diseases is multifactorial and includes a reduction 
in nutrient and calorie intake because of anorexia and dietary restrictions, impaired intestinal absorption, abnormalities of carbohydrate, lipid and protein metabolism and increased proinflammatory cytokine levels resulting in a hypermetabolic state that may occur in advanced liver disease stages [6]. Therefore for nutritional management of liver cirrhosis patients it is important to precisely assess the patient's nutritional intake and to establish effective nutritional education programs [7]. The term supportive care needs encompasses the physical, informational, emotional, practical, social and spiritual needs of an individual with chronic disease. [8]. Information need' is defined as a deficiency of information or skill related to a domain of life that is relevant to the patient. Usually patients require information concerning their disease and related care, in addition to side effects, complications, and health-related problems. It is also important to obtain information concerning additional care, daily activities, practical solutions, and financial issues. [9]. Inadequate selfmanagement skills and knowledge can lead to serious and detrimental changes in quality of life, as well as increased anxiety, distress, and difficulty coping [10]. Patient and family teaching is an important nursing role that may make the difference in the ability of the patient and family to adapt to chronic conditions .Well-informed, educated patients are more likely than uninformed patients to be concerned about their health and to do what is necessary to maintain it [11].

The aim of the study was to assess nutritional status and informational needs for patients with liver cirrhosis.

\section{SUBJECTS AND METHODS}

A descriptive exploratory design was utilized in the study. Study was conducted in tropical medicine and gastroenterology units at Zagazig University Hospitals. Field work of this study was executed in 6 months, starting from October 2016 to May 2017.

\section{Subjects:}

A purposive sample of 115 adult patients with liver cirrhosis, they were selected randomly.

Tools for data collection: three tools were used for data collection:

Tool I: A Structured interview questionnaire tool for patients was designed by the researcher after revising of related literature and opinions of expertise for content of validity and included the following three parts:

- Part 1: Demographic characteristics of patients e.g. (age, sex, marital status, occupation, level of education ....etc).

- Part 2: Nutritional profile which contain questions about follow special diet for liver cirrhosis, follow food restriction ...etc) [3].

- Part 3: Child Pugh score which consists of five items: total bilirubin, serum albumin, international normalized ratio (INR), ascites and hepatic encephalopathy [12].

Tool II: Nutritional assessment form for patients was designed by the researcher after revising of related literature and opinions of expertise for content of validity and included the following five parts.

Part 1: Subjective Global Assessment [13].

Part 2: Risk factor affecting nutritional status.

Part 3: anthropometric measurement.

Part4: Physical assessment suggestive of malnutrition [14].

Part 5: Biochemical measurements.

Tool III: Informational Needs Questionnaire [15].

\section{Content validity and Reliability:}

Content validity was used for the modified tools and the designed booklet to determine whether the tools covered the aim or not. It developed by a jury of 5 experts ,four professors from faculty of Nursing, Zagazig University and one lecturer of medical department from the Faculty of Medicine, Zagazig University, And one lecturer of Biochemistry department from the faculty of medicine. Reliability was done by using Cronbach test [16]. It was used to examine whether the subjective Global Assessment, Informational Needs Questionnaire had internal consistency or not. The test was done and the agreement percentage was $89 \%$.

\section{Administrative and Ethical considerations:}

The study was ethically approved from the dean of the faculty of Nursing, the manager of Zagazig University Hospitals, the head of tropical medicine and Gastroenterology department, ethics committee at the faculty of nursing and from Ethical committee of faculty of medicine.

\section{Statistical Design:}

All collected data were organized, categorized, tabulated, entered, and analyzed by using SPSS (Statistical Package for Social Sciences); a software program version 14, which was applied 
to frequency tables and statistical significance. The statistical significance and associations were assessed using, descriptive statistics in the form of frequencies and percentages for qualitative variables, and means and standard deviations and medians and interquartile ranges for quantitative variables, a chi-square test(X2). Spearman rank correlation.

\section{RESULTS}

The first part of our results was the Demographic characteristics and disease characteristic for patients with liver cirrhosis in the study including; gender, age, residence, marital Status, education, occupation and income (Table 1).

The second part of our results was concerned with disease characteristic; it demonstrated that The main cause of liver cirrhosis was viral hepatitis with 102 cases, studied patients were admitted to the hospital related to ascites, hematemesis and black stools (melena) $(40.0 \%$, $37.4 \%, 20.9 \%$ ) respectively). According to Child-Pugh, the highest percentage $(40.0 \%)$ of the studied patients had Child class B while (20.9\%) of them were Child class A (Table 2).

The third part of our results was concerned with nutritional status of studied patient by using different method. According to SGA, malnutrition was present in $73.9 \%$ of the patients, and of these 49 patients $(42.6 \%)$ were moderately and 36 $(31.3 \%)$ were severely malnourished, the table showed $47.8 \%$ of studied patient were under weight, according to TSF and MAC it was found $69.6 \%$ and $65.2 \%$ of studied patient suffering from malnutrition respectively (Fig. 1, Table 3).

The fourth part of our results was concerned with risk factor affecting on nutritional status, it revealed that $84.3 \%$ of the enrolled patients confirmed they had dryness of mouth, $69.5 \%$ had decreased of appetite, $60.0 \%$ inability to prepare meals (Table 4).

The fifth part of our results was concerned with laboratory assessment of the studied patients and revealed that the percentage below and above cut

off more frequent in $\mathrm{Hb}, \mathrm{PT}$, Albumin, $\mathrm{Na}$ and Ca presenting in $87.0 \%, 99.1 \%, 84.3 \%, 53.0 \%$, $80.9 \%$ ( Table 5).

The sixth part of our results was concerned with informational needs for patients; the result explains that the most important informational needs among the studied patients were medical domains $84.4 \%$. In contrast, psychological domains achieved the least important $27.0 \%$. Finally, the total informational needs arranged from moderated to high needs $33.0 \%, 67.0 \%$ respectively (Table 6).

The seventh part of our results demonstrated that there was statistical significant relation between subjective Global assessment score and patients demographic characteristics, only among their age and income in study ( $\mathrm{p}$-value $=0.01$ ), $\mathrm{p}$-value $=0.003$. . There was statistical significant relation between duration of the disease of the patients and their SGA score P-value $=0.0001$, there was highly statistically significant relation between SGA score and Child Pugh score p-value $=0.00$, there was statistically significant relations between informational needs and patient's age ( $\mathrm{P}$ - value $<0.001)$, marital status ( $\mathrm{P}$ - value $=0.009$ ), and their job $(\mathrm{P}-$ value $=0.045)$. It is noticed that the informational needs was higher among those in younger age group $(81.3 \%)$, married $(71.6 \%)$ and employees (93.8). It shows statistically significant negative correlations between total information needs score and number of abnormal signs. On the other hand, number of abnormal signs had statistically significant positive correlation with number of abnormal lab results, statistically significant negative correlation between total informational needs score and patient's age $\mathrm{r}=$ 0.520 , and Child Pugh $r=0.189$. There are also statistically significant positive correlation between SGA and age $\mathrm{r}=0.542$, duration of illness $\mathrm{r}=$ 0.478 , Child Pugh $r=0.589$, conversely there were significant negative correlation between BMI, weight deficit and SGA score (Tables 7, 8, $9,10,11,12$ ).

In multivariate analysis, indicated that the level of education, female genders were the statistically significant independent positive predictors of higher informational needs score with Standardized Coefficients $(0.24,0.25)$ respectively. Conversely, patient's age and number of abnormal signs were negative predictors with Standardized Coefficients $(-0.41 .-0.25)$ respectively, the model explains $41 \%$ of the variation in the information needs score. Age, Child Pugh and duration of illness were the statistically significant independent positive predictors of higher SGA score with Standardized Coefficients $(0.22,0.34,0.21)$ respectively. Conversely BMI was statistically significant independent negative predictors of higher SGA score with Standardized Coefficients (-0.25), the model explains $57 \%$ of the variation in the SGA scores (Tables 13, 14). 
Table (1): Personal characteristics of patients in the study sample $(n=115)$

\begin{tabular}{|c|c|c|}
\hline Personal characteristic & Frequency & Percent \\
\hline \multicolumn{3}{|l|}{ Age: } \\
\hline$<60$ & 80 & 69.6 \\
\hline $60+$ & 35 & 30.4 \\
\hline Range & \multicolumn{2}{|c|}{$26.0-65.0$} \\
\hline Mean \pm SD & \multicolumn{2}{|c|}{$53.4 \pm 9.3$} \\
\hline Median & \multicolumn{2}{|c|}{55.0} \\
\hline \multicolumn{3}{|l|}{ Gender: } \\
\hline Male & 59 & 51.3 \\
\hline Female & 56 & 48.7 \\
\hline \multicolumn{3}{|l|}{ Marital status: } \\
\hline Unmarried (single/divorced/widow) & 13 & 11.3 \\
\hline Married & 102 & 88.7 \\
\hline \multicolumn{3}{|l|}{ Job: } \\
\hline Employee & 38 & 33.0 \\
\hline Unemployed/housewife & 77 & 67.0 \\
\hline \multicolumn{3}{|l|}{ Education: } \\
\hline Uneducated & 71 & 61.7 \\
\hline Educated & 44 & 38.3 \\
\hline \multicolumn{3}{|l|}{ Residence: } \\
\hline Rural & 93 & 80.9 \\
\hline Urban & 22 & 19.1 \\
\hline \multicolumn{3}{|l|}{ Income: } \\
\hline Sufficient & 40 & 34.8 \\
\hline Insufficient & 75 & 65.2 \\
\hline
\end{tabular}


Table (2): Disease characteristics of patients in the study sample $(n=115)$

\begin{tabular}{|l|c|c|}
\hline & Frequency & Percent \\
\hline Duration of illness (years): & & 59.1 \\
\hline$<5$ & 68 & 40.9 \\
\hline $5+$ & 47 & $0.0-20.0$ \\
\hline Range & \multicolumn{2}{|c|}{$4.8 \pm 4.0$} \\
\hline Mean \pm SD & \multicolumn{2}{|c|}{4.0} \\
\hline Median & 102 & 88.7 \\
\hline Cause of liver cirrhosis: & 11 & 9.6 \\
\hline Viral & 2 & 1.7 \\
\hline Viral + schitosomiasis & \multicolumn{2}{|c|}{} \\
\hline Autoimmune & 26 & 20.9 \\
\hline Child-Pugh: & 46 & 40.0 \\
\hline A & 43 & 39.1 \\
\hline B & & 37.4 \\
\hline C & 43 & 40.0 \\
\hline Hospital admission for: & 46 & 20.9 \\
\hline Hematemesis & 24 & 13.9 \\
\hline Ascites & 16 & 10.4 \\
\hline Black stools & 12 & \\
\hline Hepatic coma & & \\
\hline General symptoms & & \\
\hline
\end{tabular}

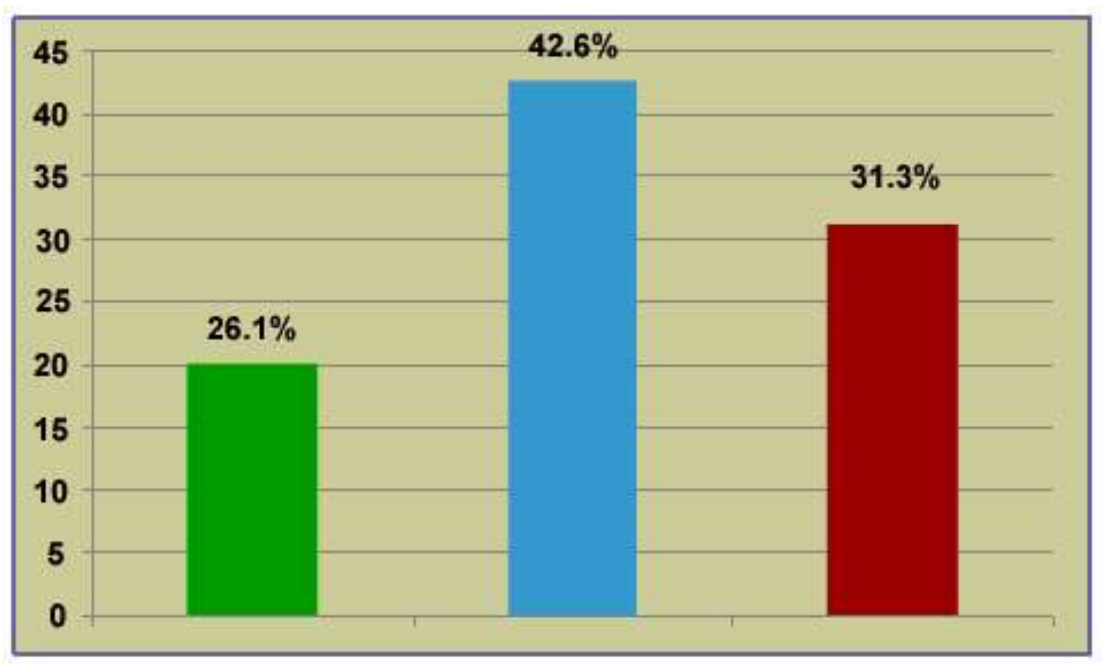

Figure (1): Nutritional status among the studied patients according to Subjective Global Assessment (SGA) score, $(\mathrm{n}=115)$ 
Table (3): Anthropometric measurements of patients in the study sample ( $\mathrm{n}=115)$

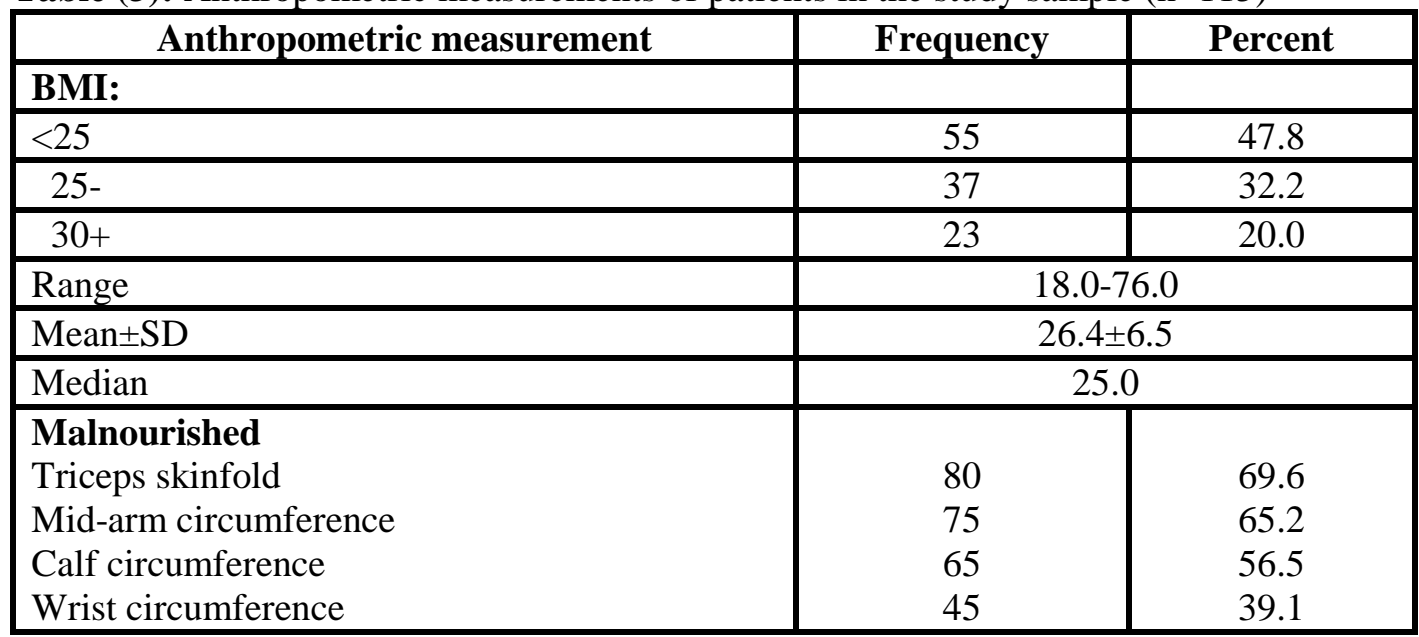

Table (4): Risk factors affecting the nutritional status among the studied patients $(n=115)$

\begin{tabular}{|l|c|c|}
\hline \multicolumn{1}{|c|}{ Risk factors } & Frequency & Percent \\
\hline Risk factors: & 97 & 84.3 \\
Dryness of mouth & 80 & 69.5 \\
Loss of appetite & 69 & 60.0 \\
Inability to prepare meals & 66 & 57.4 \\
Taste change & 58 & 50.4 \\
Mastication problems & 49 & 42.6 \\
Dyspepsia & 28 & 24.3 \\
Loneliness/ depression & 6 & 5.2 \\
Allergic & \multicolumn{2}{|c|}{$1-5$} \\
\hline No. of risk factor: & \multicolumn{2}{|c|}{$1.6 \pm 1.2$} \\
Range & \multicolumn{2}{|c|}{} \\
Mean \pm SD & \multicolumn{2}{|c|}{} \\
median & \multicolumn{2}{|c|}{} \\
\hline
\end{tabular}


Table (5): Laboratory investigation among the studied patients $(n=115)$

\begin{tabular}{|l|c|c|c|}
\hline \multicolumn{1}{|c|}{ laboratory test } & Mean(SD) & $\begin{array}{c}\text { below or above cut } \\
\text { off \% }\end{array}$ & Percent \\
\hline SGOT & $116.90 \pm(99.76)$ & $\mathrm{A}$ & 50.4 \\
\hline SGPT & $68.40 \pm(93.98)$ & $\mathrm{A}$ & 35.7 \\
\hline Albumin & $2.79 \pm(0.72)$ & $\mathrm{B}$ & 84.3 \\
\hline alkaline phosphate & $105.6 \pm(46.1)$ & $\mathrm{A}$ & 33.9 \\
\hline bilirubin(total) & $3.52 \pm(2.97)$ & $\mathrm{A}$ & 60.0 \\
\hline bilirubin(direct) & $1.82 \pm(1.34)$ & $\mathrm{A}$ & 73.0 \\
\hline prothrombin time & $18.98 \pm(3.49)$ & $\mathrm{A}$ & 99.1 \\
\hline Hemoglobin & $10.21 \pm(2.50)$ & $\mathrm{B}$ & 87.0 \\
\hline Hematocrit & $31.75 \pm(7.95)$ & $\mathrm{B}$ & 89.6 \\
\hline Sodium & $132.65 \pm(7.36)$ & $\mathrm{B}$ & 53.0 \\
\hline Potassium & $3.73 \pm(0.94)$ & $\mathrm{N}$ & 60.0 \\
\hline Calcium & $7.62 \pm(1.22)$ & $\mathrm{B}$ & 80.9 \\
\hline Cholesterol & $106.0 \pm(36.7)$ & $\mathrm{B}$ & 67.8 \\
\hline Phosphate & $4.1 \pm(1.2)$ & $\mathrm{N}$ & 70.4 \\
\hline Wbcs & $7.31 \pm(3.19)$ & $\mathrm{N}$ & 72.2 \\
\hline $\begin{array}{l}\text { No. of lab anomalies: } \\
\text { range } \\
\text { mean } \pm \text { sd } \\
\text { median }\end{array}$ & & $1-13$ & \\
\hline
\end{tabular}

Table (6): information needs among the studied patients ( $\mathrm{n}=115)$

\begin{tabular}{|c|c|c|}
\hline Information needs & Frequency & Percent \\
\hline Medical domain : & & \\
\hline Low & 2 & 1.7 \\
\hline Moderate & 16 & 13.9 \\
\hline High & 97 & 84.4 \\
\hline Treatment: & & \\
\hline Low & 8 & 7.0 \\
\hline Moderate & 21 & 18.3 \\
\hline High & 86 & 74.7 \\
\hline Investigations: & & \\
\hline Low & 12 & 10.4 \\
\hline Moderate & 46 & 40.0 \\
\hline High & 57 & 49.6 \\
\hline Physical domain: & & \\
\hline Low & 4 & 3.5 \\
\hline Moderate & 44 & 38.3 \\
\hline High & 67 & 58.3 \\
\hline Psychological domain : & & \\
\hline Low & 49 & 42.6 \\
\hline Moderate & 35 & 30.4 \\
\hline High & 31 & 27.0 \\
\hline
\end{tabular}


Table (7): Relation between SGA score among the studied patients and their personal characteristics

\begin{tabular}{|l|c|c|c|c|c|c|c|c|}
\hline \multirow{3}{*}{ Personal characteristics } & \multicolumn{6}{|c|}{$\begin{array}{c}\text { Good } \\
\text { nutrition }\end{array}$} & $\begin{array}{c}\text { Moderated } \\
\text { malnutrition }\end{array}$ & \multicolumn{2}{c|}{$\begin{array}{c}\text { Severe } \\
\text { malnutrition }\end{array}$} & \multirow{2}{*}{ X $^{2}$} & \multirow{2}{*}{$\begin{array}{c}\text { p- } \\
\text { value }\end{array}$} \\
\cline { 2 - 9 } & No & \% & No & \% & No & \% & & \\
\hline Age: & & & & & & & & \\
\hline$<60$ & 26 & 32.5 & 35 & 43.7 & 19 & 23.8 & & \\
\hline 60+ & 4 & 11.4 & 14 & 40.0 & 17 & 48.6 & 9.01 & $0.01^{*}$ \\
\hline Gender: & & & & & & & & \\
\hline Male & 15 & 25.4 & 26 & 44.1 & 18 & 30.5 & 0.1 & 0.94 \\
\hline Female & 15 & 26.8 & 23 & 41.1 & 18 & 32.1 & & \\
\hline Marital status: & & & & & & & & \\
\hline Unmarried & 3 & 23.0 & 6 & 46.2 & 4 & 30.8 & 0.09 & 0.35 \\
\hline Married & 27 & 26.5 & 43 & 42.2 & 32 & 31.3 & & \\
\hline Job: & & & & & & & & \\
\hline Worker & 15 & 39.5 & 15 & 39.5 & 8 & 21 & 5.9 & 0.061 \\
\hline Unemployed/housewife & 15 & 19.5 & 34 & 44.1 & 28 & 36.4 & & \\
\hline Education: & & & & & & & & \\
\hline Illiterate & 21 & 29.6 & 29 & 40.8 & 21 & 29.6 & & \\
\hline Basic & 5 & 21.8 & 11 & 47.8 & 7 & 30.4 & 1.47 & 0.83 \\
\hline Secondary & 4 & 19 & 9 & 42.9 & 8 & 38.1 & & \\
\hline Residence: & & & & & & & & \\
\hline Rural & 22 & 23.7 & 39 & 41.9 & 32 & 34.4 & 2.65 & 0.26 \\
\hline Urban & 8 & 36.3 & 10 & 45.5 & 4 & 18.2 & & \\
\hline Income: & & & & & & & & \\
\hline Sufficient & 10 & 25 & 18 & 45 & 12 & 30 & & \\
\hline Insufficient & 20 & 26.7 & 31 & 41.3 & 24 & 32 & 8.60 & $0.003^{*}$ \\
\hline
\end{tabular}

(*) statistically significant at $\mathbf{p}<0.05$

(**) statistically significant at $\mathbf{p}<0.01$

Table (8): Relation between SGA score among the studied patients and their disease characteristics

\begin{tabular}{|c|c|c|c|c|c|c|c|c|}
\hline \multirow{3}{*}{ Disease characteristics } & \multicolumn{6}{|c|}{ Subjective Global assessment } & \multirow{3}{*}{$\mathrm{X}^{2}$ test } & \multirow{3}{*}{ p-value } \\
\hline & \multicolumn{2}{|c|}{$\begin{array}{c}\text { Good } \\
\text { nutrition }\end{array}$} & \multicolumn{2}{|c|}{$\begin{array}{c}\text { Moderated } \\
\text { malnutrition }\end{array}$} & \multicolumn{2}{|c|}{$\begin{array}{c}\text { Severe } \\
\text { malnutrition }\end{array}$} & & \\
\hline & No & $\%$ & No & $\%$ & No & $\%$ & & \\
\hline \multicolumn{9}{|l|}{ Duration of illness (years): } \\
\hline$<5$ & 29 & 42.6 & 25 & 36.8 & 14 & 20.6 & & \\
\hline $5+$ & 1 & 2.1 & 24 & 51.1 & 22 & 46.8 & 17.7 & $0.0001 * *$ \\
\hline \multirow{2}{*}{$\begin{array}{l}\text { Child-Pugh: } \\
\text { A }\end{array}$} & & & & & & & & \\
\hline & 19 & 73.1 & 3 & 11.5 & 4 & 15.4 & & \\
\hline $\mathrm{B}$ & 5 & 10.9 & 29 & 63.0 & 12 & 26.1 & Fisher & $0.00 * *$ \\
\hline $\mathrm{C}$ & 6 & 14 & 17 & 39.5 & 20 & 46.5 & & \\
\hline
\end{tabular}

(*) statistically significant at $\mathbf{p}<0.05$

(**) statistically significant at $\mathbf{p}<0.01$ 
Table (9): Relations between patient's information needs and their personal characteristics

\begin{tabular}{|c|c|c|c|c|c|c|}
\hline \multirow{3}{*}{ Personal characteristic } & \multicolumn{4}{|c|}{ Needs } & \multirow{3}{*}{$\mathrm{X}^{2}$ test } & \multirow{3}{*}{ p-value } \\
\hline & \multicolumn{2}{|c|}{ Moderate } & \multicolumn{2}{|c|}{ High } & & \\
\hline & No. & $\%$ & No. & $\%$ & & \\
\hline \multicolumn{7}{|l|}{ Age: } \\
\hline$<60$ & 15 & 18.8 & 65 & 81.3 & & \\
\hline $60+$ & 23 & 65.7 & 12 & 34.3 & 24.27 & $<0.001^{*}$ \\
\hline \multicolumn{7}{|l|}{ Gender: } \\
\hline Male & 23 & 39.0 & 36 & 61.0 & & \\
\hline Female & 15 & 26.8 & 41 & 73.2 & 1.93 & 0.16 \\
\hline \multicolumn{7}{|l|}{ Marital status: } \\
\hline Unmarried (single/divorced/widow) & 9 & 69.2 & 4 & 30.8 & & \\
\hline Married & 29 & 28.4 & 73 & 71.6 & Fisher & $0.009^{*}$ \\
\hline \multicolumn{7}{|l|}{ Job: } \\
\hline Employee & 1 & 6.3 & 15 & 93.8 & & \\
\hline Worker & 9 & 40.9 & 13 & 59.1 & 6.19 & $0.045^{*}$ \\
\hline Unemployed/housewife & 28 & 36.4 & 49 & 63.6 & & \\
\hline \multicolumn{7}{|l|}{ Education: } \\
\hline Illiterate & 28 & 39.4 & 43 & 60.6 & & \\
\hline Basic & 7 & 30.4 & 16 & 69.6 & 4.72 & 0.09 \\
\hline Secondary & 3 & 14.3 & 18 & 85.7 & & \\
\hline \multicolumn{7}{|l|}{ Residence: } \\
\hline Rural & 32 & 34.4 & 61 & 65.6 & & \\
\hline Urban & 6 & 27.3 & 16 & 72.7 & 0.41 & 0.52 \\
\hline \multicolumn{7}{|l|}{ Income: } \\
\hline Sufficient & 11 & 27.5 & 29 & 72.5 & & \\
\hline Insufficient & 27 & 36.0 & 48 & 64.0 & 0.85 & 0.36 \\
\hline
\end{tabular}

(*) statistically significant at $\mathbf{p}<0.05$

(**) statistically significant at $\mathbf{p}<0.01$

Table (10): Relations between patient's information needs and their disease characteristics and BMI

\begin{tabular}{|c|c|c|c|c|c|c|}
\hline \multirow{3}{*}{ Patient's characteristics } & \multicolumn{4}{|c|}{ Needs } & \multirow{3}{*}{$\mathrm{X}^{2}$ test } & \multirow{3}{*}{ p-value } \\
\hline & \multicolumn{2}{|c|}{ Moderate } & \multicolumn{2}{|c|}{ High } & & \\
\hline & No. & $\%$ & No. & $\%$ & & \\
\hline \multicolumn{7}{|l|}{ Duration of illness (years): } \\
\hline$<5$ & 21 & 30.9 & 47 & 69.1 & & \\
\hline $5+$ & 17 & 36.2 & 30 & 63.8 & 0.35 & 0.55 \\
\hline \multicolumn{7}{|l|}{ Child-Pugh: } \\
\hline $\mathrm{A}$ & 3 & 11.5 & 23 & 88.5 & & \\
\hline $\mathrm{B}$ & 24 & 51.2 & 22 & 47.8 & 1.22 & 0.54 \\
\hline $\mathrm{C}$ & 11 & 25.6 & 32 & 74.4 & & \\
\hline \multicolumn{7}{|l|}{ BMI: } \\
\hline Under weight & 22 & 40.0 & 33 & 60.0 & & \\
\hline Over weight & 11 & 29.7 & 26 & 70.3 & 2.72 & 0.26 \\
\hline Obese & 5 & 21. & 18 & 78.3 & & \\
\hline
\end{tabular}

(*) statistically significant at $\mathbf{p}<0.05$

(**) statistically significant at $\mathbf{p}<0.01$ 
Table (11): Correlation matrix of needs score, number of abnormal signs and abnormal lab findings

\begin{tabular}{|l|c|c|c|}
\hline \multirow{2}{*}{\multicolumn{1}{|c|}{ Item }} & \multicolumn{3}{c|}{ Spearman's rank correlation coefficient } \\
\cline { 2 - 4 } & Needs score & No. of abnormal signs & No. of abnormal lab \\
\hline Needs score & & & \\
\hline No. of abnormal signs & $-.366^{* *}$ & & \\
\hline No. of abnormal lab & -0.11 & $.198^{*}$ & \\
\hline
\end{tabular}

$(*)$ statistically significant at $\mathbf{p}<0.05$

(**) statistically significant at $\mathbf{p}<0.01$

Table (12): Correlation matrix of needs score, SGA score, number of abnormal signs and abnormal lab findings and patient's characteristics

\begin{tabular}{|l|c|c|c|c|}
\hline \multirow{2}{*}{\multicolumn{1}{|c|}{ Item }} & \multicolumn{4}{|c|}{ Spearman's rank correlation coefficient } \\
\cline { 2 - 5 } & Needs score & SGA score & No. of abnormal signs & No. of abnormal lab \\
\hline Age & $-.520^{* *}$ & $0.542^{* *}$ & $.284^{* *}$ & 0.08 \\
\hline Education & $.304 * *$ & 0.45 & $-.339^{* *}$ & 0.00 \\
\hline Duration of illness & -0.18 & $0.478^{* *}$ & $.263^{* *}$ & 0.03 \\
\hline Child-Pugh & $-.189 *$ & $0.589^{* *}$ & $.313^{* *}$ & $.437 * *$ \\
\hline Weight deficit & 0.15 & $-0.421^{* *}$ & 0.02 & 0.03 \\
\hline BMI & 0.14 & $-0.342^{* *}$ & 0.02 & 0.03 \\
\hline
\end{tabular}

$\left.{ }^{*}\right)$ statistically significant at $p<0.05$

$(* *)$ statistically significant at $\mathbf{p}<0.01$

Table (13): Best fitting multiple linear regression models for the Information Needs Score

\begin{tabular}{|l|c|c|c|c|c|c|c|}
\hline \multirow{2}{*}{ Item } & \multicolumn{2}{|c|}{$\begin{array}{c}\text { Unstandardized } \\
\text { Coefficients }\end{array}$} & \multirow{2}{*}{$\begin{array}{c}\text { Standardized } \\
\text { Coefficients }\end{array}$} & \multirow{2}{*}{ t-test } & \multirow{2}{*}{ p-value } & \multicolumn{2}{|c|}{$\begin{array}{c}\text { 95\% Confidence } \\
\text { Interval for B }\end{array}$} \\
\cline { 2 - 5 } & B & Std. Error & & & & Lower & Upper \\
\hline Constant & 90.83 & 6.66 & & 13.636 & $<0.001$ & 77.63 & 104.03 \\
\hline Age & -0.44 & 0.08 & -0.41 & -5.219 & $<0.001$ & -0.60 & -0.27 \\
\hline Female gender & 5.06 & 1.64 & 0.25 & 3.087 & 0.003 & 1.81 & 8.31 \\
\hline Education & 3.00 & 1.10 & 0.24 & 2.730 & 0.007 & 0.82 & 5.18 \\
\hline No. of abnormal signs & -1.56 & 0.50 & -0.25 & -3.113 & 0.002 & -2.56 & -0.57 \\
\hline
\end{tabular}

Table 14: Best fitting multiple linear regression models for the Subjective Global Assessment score

\begin{tabular}{|l|c|c|c|c|c|c|c|}
\hline \multirow{2}{*}{ Item } & \multicolumn{2}{|c|}{$\begin{array}{c}\text { Unstandardized } \\
\text { Coefficients }\end{array}$} & \multirow{2}{*}{$\begin{array}{c}\text { Standardized } \\
\text { Coefficients }\end{array}$} & \multirow{2}{*}{ t-test } & \multirow{2}{*}{ p-value } & \multicolumn{2}{c|}{$\begin{array}{c}\text { 95\% Confidence } \\
\text { Interval for B }\end{array}$} \\
\cline { 2 - 5 } & B & Std. Error & & & & Lower & Upper \\
\hline Constant & 76.43 & 7.56 & & 8.355 & $<0.001$ & 66.98 & 99.89 \\
\hline Age & 6.25 & 3.35 & 0.22 & 2.727 & 0.007 & 2.55 & 15.96 \\
\hline Child pugh & 1.25 & 0.27 & 0.34 & 4.334 & $<0.001$ & 0.69 & 1.83 \\
\hline BMI & -9.81 & 3.09 & -0.25 & -3.198 & 0.006 & -16.80 & -3.74 \\
\hline Duration of illness & 4.18 & 1.51 & 0.21 & 3.756 & 0.001 & 0.65 & 1.87 \\
\hline
\end{tabular}




\section{DISCUSSION}

Liver cirrhosis is the terminal stage in the natural history of chronic liver diseases [17]. Malnutrition is very common in liver disease and gets worse with the severity of the underlying liver problems [18]. Malnutrition is defined as clinical and biochemical alteration due to a primary or secondary deficit of nutrients in the daily food intake of a person and it has a high impact on quality of life and on morbidity and mortality [19]. Complete and reliable information is important to them both during and after treatment. It assists patients in making treatment decisions, managing immediate effects of treatment, and reducing feelings of vulnerability. It can also increase health competence and give patients a sense of control over the illness [20].

Discussion of the results will cover these areas in the following sequence; demographic characteristics and diseases characteristic of adult patients with liver cirrhosis under the study, nutritional status among studied patients by using different methods, informational needs of patients with liver cirrhosis and relation and correlation between different variables.

Demographic characteristics and disease characteristic for patients with liver cirrhosis in the study including; gender, age, residence, marital status, education, occupation and income were matched and this help to control other variables that may affect the nutritional status and informational needs for patients with liver cirrhosis in the study, The present study classified Child Pugh into three groups Child A, B, C: in the present study indicated that Child $\mathrm{P}$ values were more than Child $\mathrm{C}$ values $(40 \%, 39.1 \%)$ respectively. In the present study, we evaluated the nutritional status of the cirrhotic by different methods and it was observed that subjective global assessment (SGA) diagnosed $73.9 \%$ of patients as malnutrition. The current study result was congruent with the finding of an Egyptian study conducted in Assiut by Khalil [21] ,Romeiro and Augusti [22], Bakshi and Singh, [3], Vieira [23], Salah [24]. Campillo [25] proposed different BMI cut-off values for patients with liver cirrhosis depending on the presence and severity of ascites. The present study revealed that near of half of studied patients had BMI under $25 \mathrm{~kg} / \mathrm{m} 2$, this study in line with Bakshi and Singh[3] in India that found more than one third of studied sample (37.2) suffering from malnutrition by BMI.
Among the anthropometric methods used, \%TSF was the one that most frequently diagnosed malnutrition (69.9\%). Although it is theoretically possible that the presence of swelling could hide a depletion in adipose tissue and that the frequency of diagnosis of malnutrition could be reduced by the $\%$ TSF method, it has been reported that in patients with chronic liver disease, the upper limbs are not the preferential place of swelling, similar finding was reported by Vieira [23] and Monsef [26]. The present study revealed that; the most common risk factors of malnutrition are dry mouth, loss of appetite, taste alteration, inability to prepare meals, This results partially agree with finding of, Khalil [21] and Monsef [26] who found that the majority of studied patients had loss of appetite, dryness of mouth , and reported that; multiple factors which are common to the underlying disease directly contribute to malnutrition, among them.

Regarding laboratory investigation: Serum albumin concentration is the most frequently used laboratory measure of nutritional status, the present study revealed that more than three quarter of studied patient have reduction of serum albumin with mean $2.79 \pm 0.72$ on the same line Figueiredo [27] in Brazil who found decreased in serum albumin with mean $2.7 \pm 0.4$ Also Vieira [23] who found that Serum albumin was reduced in more than half of studied patient and report that The reduction of serum albumin levels in HC patients, principally among those with moderate or severe hepatic insufficiency, could be associated with either malnutrition, due to reduction in food intake and to the worsening metabolism of nutrients, or with the hepatic dysfunction itself which compromises albumin .

Regarding Hemoglobin the present study revealed that the mean value of hemoglobin was decreased $10.21 \pm 2.5$ on the same line Monsef [26] and Dataller and Gines [28] who pointed out that anemia is a common manifestation of liver cirrhosis; it may be due to gastrointestinal bleeding, likewise Merli [29] who found that the mean value of hemoglobin was $(12.9 \pm 1.8)$. This result may be due to difference in severity of diseases in studied patient, regarding informational needs. The present study findings revealed that patients placed the greatest importance on needing information in the medical domain. This result is similar to other studies that have examined informational needs of patients with liver cirrhosis as Ko [30], Volk [3], Ng [31], Burnham [32] and Gillespie [10] who found that the highest need 
for information reported by patients in their study was related to medical domain .The patients in this study expressed a need for information, understanding, and education about liver cirrhosis and possible complications to selfmanage their disease and maintain their health. Likewise Papadakos [33] who found that patients considered medical information less important because of the knowledge and experience they have gained over the course of their illness.

Although the need for medical information was the greatest, the informational needs regard treatment domain had the second highest importance scores associated with it. This study in line with Alizadeh [34] and Fabris [35] who found that concerns about the need for specific treatment was reported by majority of studied patients as side effects of treatment and proper medications, this due to the majority of studied patients not received any informational needs regarding treatment from nurse specialist. And disagree with Grogan and Timmins [36] and Minuk [37] who that found only (11\%, $23 \%$ respectively) of studied patients needed information regarding treatment, this due to patient were eager to receive information on treatment.

The next most highly rated domain of need is the physical domain with high score as controlling/ reducing abdominal distention, ways of controlling symptoms (fatigue, pruritus) this was similar to other studies of hepatic patients as Zandi [38] who found that managing side effects, including abdominal distension, fatigue and tiredness, were among the highest rated information needs.

The psychological domain received the lowest importance score, only $27.0 \%$ and this is consistent with Chen [39] and Janke [40] that found studied patients had low score of informational needs regard psychological domain likewise with Conrad [41] and jessop (42) found that psychological domain had highest important score in informational needs among studied patients .This difference perhaps due to the patient perception that this domain is out of the

As regard to the relation between patients' age \& SGA, the present study results indicates that, there was a statistically significant relation between age and SGA score of malnutrition $(\mathrm{P}=0.01)$, this is supported by the finding that, there was a statistically positive correlation between SGA score and age. This finding should be taken cautiously as may confound the relation between liver affection and nutritional status of those patients, because increasing age of the patient is usually associated with high prevalence of geriatric problem that usually affect their nutritional status and may predispose to malnutrition as anorexia, dental problem, this result on same line with Salah [24] .Regarding Child- Pugh and its relation with SGA score, the present study revealed that there was a statistical significant relationship between Child- Pugh and SGA, this is further supported by the finding that, there was a statistically significant positive correlation between Child Pugh and SGA. From these results we can observe a trend towards a higher proportion of bad nutritional status in patients with Child-Pugh B \& C compared to Child-Pugh A, this indicates that SGA tool has the ability to reflect the degree of severity of liver affection in the study sample. The current study revealed that there was a statistically significant relationship between informational needs and studied patient age $p=0.001$, this is further supported by the finding that, there was a statistically significant negative correlation between total informational needs and patient with younger age $r=.520$, these findings might be due to the younger age at the beginning of his life need more information to self- manage their diseases. These findings in accordance with previous studies, Papadakos [33], and with Hassan and Shams [43] which demonstrated that younger patients placed more importance on the informational needs when compared to older patients . Likewise other study by Dehghani [44] who found that, there was statistically significance positive relation between informational needs and older age ,these finding might be due to that old age predicted higher unmet needs.

Apart from total abnormal signs, the current study revealed that, there was a statistically significant negative correlation between number of abnormal signs, and informational needs. The negative relation between number of abnormal signs, and needs was confirmed in multivariate analysis.

On summary, Malnutrition is highly prevalent among the patients with liver cirrhosis, it varied according to the method used. The most prioritized informational needs for patients with liver cirrhosis was medical domain whereas the least priority was given to the psychological domain. It also shows that information needs differ based on some socio-demographic and clinical characteristics and physical condition. 


\section{Funding: None. \\ Conflicts of interest: None. \\ Ethical approval:Approved .}

\section{REFERENCES}

1- Amin, H, Samie R, Hamed F, Abo Zaid E, Hammad E. Assessment of Nutritional Status of Patients with Chronic Hepatitis C and HCV-Related Cirrhosis in the Compensated Stage, American Journal of Internal Medicine; 2016 4(2): 24-35.

2- Volk M, Fisher N, Fontana R. Patient knowledge about disease self-management in cirrhosis. Am J Gastroenterol; 2013, 108 (3):302-305.

3- Bakshi N, Singh K. Nutrition assessment and its effect on various clinical variables among patients undergoing liver transplant HepatoBiliary Surg Nutr; 2016, 5(4):358-371.

4- Huisman E, Trip J, Siersema P, van Hoek B, van Erpecum K. Protein energy malnutrition predicts complications in liver cirrhosis. Eur J Gastroenterol Hepatol; 2013, 23: 982-989.

5- Nunes F, Bassani L, Fernandes S, Deutrich M, Pivatto B, Marroni C. food consumption of cirrhotic patients, comparison with the nutritional status and disease staging: 2016,53 (4) 250-257.

6- Merli M, Giusto M, Gentili F, Novelli G, Ferretti $\mathrm{G}$, Riggio $\mathrm{O}$ et al. Nutritional status: it's influence on the outcome of patients undergoing liver transplantation, Liver International Journal, 2010, 30( 2 ) 208-214.

7- Yasutake K, Bekki M, Ichinose M, Ikemoto M, Fujino T, Ryu T et al. Assessing current nutritional status of patients with HCV-related liver cirrhosis in the compensated stage. Asia Pac J Clin Nutr; 2012, 21 (3): 400-405.

8- Valery P, Powell E, Moses N, Volk M, Mcphail S, Clark P. unmet supportive care needs in people diagnosed with chronic liver disease .BMJ ;2015.Available at: http://bmjopen. Bmj.com .Access at: $1 / 2 / 2016$.

9- Mendes K, Junior O, Ziviani L, Rossin F, Zago M, Galvão C.: Educational intervention for liver transplantation candidates Rev. Latino-Am. Enfermagem: 2013, 21 (1) 25-36.

10- Gillespie J, Kacikanis A, Nyhof-Young J, Gallinger S, Ruthig E. Information Needs of Hepato-Pancreato-Biliary Surgica Oncology Patients. J Canc Edu: 2016, 32 (3):589-595.

11- Mahmoud B, Shafik N, Attya S . Impact of a designed supportive nursing program for hepatitis $\mathrm{C}$ patients on their functional health status during interferon therapy in the national hepatology medicine institute. Nat Sci: 2013, 11(6):80-90.
12- Pugh R, Murray-Lyon I, Dawson J . Transsection of the oesophagus for bleeding oesophageal varices. Br J Surg: 1973 (60) 646-649.

13- Detsky A, McLaughlin J, Baker J, Johnston N, Whittaker S, Mendelson R and Jeejeebhoy, K. What Is Subjective Global Assessment of Nutritional Status? JPEN, 1987 (11), 8-13.

14- Jarvis c. Physical Examination \& Health Assessment, Nutrition and metabolic assessment: 7 Edition, 2015, pp 530-531 Elsevier-Health Sciences Division.

15- Galloway S, Graydon J , Harrison D, Evans-Boyden B , Palmer-Wickham $\mathrm{S}$ et al. Informational needs of women with a recent diagnosis of breast cancer: development and initial testing of a tool. Journal of Advanced Nursing, 1997 (25), 11751183.

16- Hasse J, Strong S, Gorman M, Liepa G. Subjective global assessment: alternative nutrition-assessment technique for liver-transplant candidates.Nutrition; 1993 9(4):339-343.

17- Anthony L, Escheik C, Gerber N, Younossi Z. Quality of life in cirrhosis: Current Gastroenterol Rep, 2013 (15) p. 301.

18- Sidiq T, Khan N. Nutrition as a Part of Therapy in the Treatment of Liver Cirrhosis. Nutrition \& Food Sciences Tahira, J Nutr Food Science: 2013, 5(11) 24-32.

19- Baraldi C, De Ruvo N, Iemmolo R, Ballarin, R, Cautero N, Gerunda G , Di Benedetto F.Relevance of the Nutritional Care in Patients before Liver Transplantation, Global Journal of Gastroenterology \& Hepatology, 2014, (2), 92-100.

20- Rutten L, Arora N, Bakos A, Aziz N, Rowland J.; Information needs and sources of information among cancer patients: a systematic review of research, Patient Educ Couns: 2005, 57(3):250-61.

21- Khalil S, Youssef M, Mekkawy M, Abdelmalek M. Liver Cirrhosis: Impact Of Nutritional Regimen On Patients Outcome 2015, 4 ( 2) 22-35.

22- Romeiro F, Augusti L. Nutritional assessment in cirrhotic patients with hepatic encephalopath World J Hepatol; 2015, 7(30): 2940-2954.

23- Vieira P, Daurea A, De-Souza D, Oliveira L. Nutritional assessment in hepatic cirrhosis; clinical, anthropometric, biochemical and hematological parameters, Nutr Hosp, 2013 ;28(5):1615-1621.

24- Saleh M, Mustafa H, Eid K, Soliman M , Sultan $\mathrm{H}$. assessment of nutritional status of patients with chronic liver diseases admitted to gastroenterology department al-azhar university hospital, Assiut. AAMJ, 2014, 12 (3): 245-249. 
25- Campillo B, Richardet J, Bories P. Validation of Body Mass Index for the Diagnosis of Malnutrition in Patients with Liver Cirrhosis. Gastroentérologie Clinique et Biologique, 2006 (30): 1137-1143.

26- Monsef W, Mostafa I., Zaky D.: Assessment of the Nutritional Status of the Egyptian Patient with End Stage Liver Disease Prior to Liver Transplantation Open Journal of Gastroenterology 2014, 4: 159-169

27- Figueiredo F, Perez R, Freitas M, Kondo, M. Comparison of three methods of nutritional assessment in liver cirrhosis: subjective global assessment, traditional nutritional parameters, and body composition analysis, J Gastroenterol: 2009, 41(5) : 476-482.

28- Datalle R , Gines P. Cirrhosis of the liver, available at http://www.emedicine.com/med/ topic2/76.htm accessed on 14 March at $2.30 \mathrm{Am}$ 2017.

29- Merli M, Giusto M, Gentili F, Novelli G, Ferretti G, Riggio $\mathrm{O}$ et al. Nutritional status: it's influence on the outcome of patients undergoing liver transplantation, Liver International Journal : 2010, 30( 2 ) 208-214.

30 - Ko D, Lee I, Muehrer R. Informational needs of liver transplant recipients during a two year post transplant period Chronic Ill n. 2016, 12(1): 2940.

31- Ng C, Low W, Wong L. Uncovering the experiences and needs of patients with chronic hepatitis B infection at diagnosis:a qualitative study. Asia Pac J Public Health; 2013, 25(12): 32-40.

32- Burnham B, Wallington S, Jillson A . Knowledge, attitudes, and beliefs of patients with chronic liver disease. Am J Health Behavior; 2014, 38: 737- 744.

33. Papadakos J, Urowitz S, Olmstead C, Friedman $\mathrm{A}$, Zhu J, Catton P. Informational needs of gastrointestinal oncology patients John Wiley \& Sons Ltd Health Expectations, 2014, (18):30883098.
34. Alizadeh A, Ranjbar M, Yadollahzadeh, M. Patient concerns regarding chronic hepatitis B and C infection. East Mediterr Health J; 2008, 14(5): 1142-1147.

35- Fabris P, Tositti G, Giordani M. Assessing patients' understanding of hepatitis $\mathrm{C}$ virus infection and its impact on their lifestyle. Aliment Pharmacol Ther; 2006, 23:1161-1170.

36- Grogan A, Timmins F. Patients' perceptions of information and support received from the nurse specialist during HCV treatment. J Clin Nurs; 2010, (19): 2869-2878.

37- Minuk G, Gutkin A, Wong C. Patient concerns regarding chronic hepatitis $\mathrm{C}$ infections. $J$ Viral Hepat; 2005 (12): 51-57.

38- Zandi M, Hajbagheri M, Memarian R, Nejhad A, Alavian S. Effects of a self-care program on quality of life of cirrhotic patients referring to Tehran Hepatitis Center Health and Quality of Life Outcomes, 2005: 3(35) : 256- 270.

39- Chen M, Hung H, Chang H, Yang S, Tsai, W, Chen C. Assessment of Educational Needs and Quality of Life of Chronic Hepatitis Patients: BMC Health Services Research, 2017 (17): p: 148.

40- Janke E, McGraw S, Garcia-Tsao G. Psychosocial issues in hepatitis $\mathrm{C}$ : a qualitative analysis. Psychosomatic, 2008, 49: 494-501.

41- Conrad S, Garrett L, Cooksley W. Living with chronic hepatitis $\mathrm{C}$ means 'you just haven't got a normal life any more'. Chronic Illn : 2006 (2): 121-131.

42- Jessop A, Cohen C, Burke M . Hepatitis support groups: meeting the information and support needs of hepatitis patients. Gastroenterol Nurs; 2004, 27: 163-169.

43-Hassan M, Shams R. awareness of viral hepatitis (b \& c) among attendance of viral hepatitis Clinic-Assiut University Hospital. Am J, 2009, 7 ( 3) 185- 197.

44. Dehghani H, Dehghani K, Emami-Mybodi, M, Rahimianfar A, Soltani H. Physical and Psychosocial Needs Assessment in Patients with Heart Failure in the Yazd Afshar Hospital J. Clie. Care, 2017, 1 (1): 1-5. 